\title{
Pseudarthrosen und schlecht heilende Frakturen am Humerusschaft und am distalen Humerus
}

Michael Plecko

\section{Einleitung}

Frakturen des Humerusschaftes machen ca. 1-3\% aller frischen Frakturen und etwa 20-30\% der Frakturen des Oberarmknochens aus, während die Frakturen des distalen Humerus ungefähr $2 \%$ aller Frakturen ausmachen. Beide Verletzungen weisen eine bimodale Altersverteilung auf. Während die Schaftfrakturen nicht selten konservativ, meist mit einem Brace nach Sarmiento et al. versorgt werden [1,2], steht bei den distalen Frakturen in der Akutbehandlung die operative Versorgung mit offener Osteosynthese im Vordergrund [3]. Die Ursachen einer verzögerten oder ausbleibenden Frakturheilung können vielfältig sein und oft handelt es sich um mehrere Faktoren, die hier zusammenspielen. Allgemein spricht man von einer Pseudarthrose, wenn nach 24 Wochen (6 Monaten) noch keine knöcherne Konsolidierung eingetreten ist [4]. Während Pseudarthrosen im Humerusschaft zu erheblichen Beschwerden und Funktionseinschränkungen führen, werden Pseudarthrosen am distalen Humerus oft erstaunlich lange gut toleriert. Periartikuläre Pseudarthrosen sind auch meist mit erheblichen Gelenkkontrakturen vergesellschaftet.

\section{Humerusschaft}

Die Häufigkeit einer verzögerten oder ausbleibenden Heilung hängt beim Humerusschaft ab von der Frakturform (Querfraktur, kurze Schrägfraktur, Spiralfraktur, Segmentfraktur, Trümmerfraktur, Defektfraktur), von der Lokalisation der Fraktur (proximales, mittleres, distales Drittel), ob es sich um eine Fraktur mit Weichteilschaden oder gar eine offene Fraktur handelt, und von Kofaktoren wie hohem Alter, Nikotinabusus oder der Einnahme von bestimmten Medikamenten wie z.B. nicht steroidalen Antirheumatika. Die Inzidenz von Pseudarthrosen beträgt im proximalen Schaftdrittel $24 \%$, im mittleren Drittel 12\% und im distalen Schaftdrittel 15\% [5]. Obgleich manche Studien bei frischen Schaftfrakturen eine ähnliche Ausheilungsrate und Ausheilungszeit für konservativ behandelte PatientInnen im Vergleich zu operativ versorgten PatientInnen angeben, gibt es doch eine gewisse Evidenz, dass die Osteosynthese (Plattenosteosynthese) zu einer deutlich geringeren Rate an Fehlverheilungen und Pseudarthrosen führt $[6,7]$. Auch im eigenen Krankengut können die hohen Ausheilungsraten, die von Sarmiento et al. [1] angegeben wurden, mit der Brace-Behandlung nicht erreicht werden. Dies deckt sich auch mit der Erfahrung anderer Zentren. So wurden von verschiedenen Autoren hohe Pseudarthrosenraten nach konservativer Behandlung zwischen 5,5 und 33\% angegeben [8, 9] ( Abb. 1). Hochenergetische Traumen, Knochenverlust, Weichteilinterposition sowie eine unzureichende Reposition mit mangelnder Annäherung der Frakturenden, aber auch eine vorbestehende Kontraktur der benachbarten Gelenke und dadurch vermehrte Bewegung in der Fraktur begünstigen zusätzlich die Entstehung einer Pseudarthrose.

\section{Behandlung}

Bevor Entscheidungen über die definitive Behandlung einer Humeruspseudarthrose getroffen werden können, ist neben der Beurteilung, ob es sich um eine vitale, biologisch aktive Pseudarthrose handelt oder ob keine biologische Aktivität vorliegt, eine Einschätzung über etwaige Knochendefekte zu treffen und eine gründliche Ursachenerforschung notwendig. Es ist zu ergründen, ob ein vorwiegend mechanisches Problem (z. B. zu große Instabilität oder unzureichende Reposition) zum Ausbleiben einer zeitgerechten Heilung geführt hat oder ob biologische Faktoren (z.B. verminderte Zellaktivität, lokale Durchblutung) ursächlich für die Pseudarthrosenentstehung waren. Entsprechend dem Diamond-Konzept wird dann ein für die Einzelsituation optimiertes Behandlungskonzept erstellt, um die größtmögliche Erfolgswahrscheinlichkeit zu erzielen $[10,11]$. Von großer Bedeutung ist auch der präoperativ oft schwierige Ausschluss einer "low-grade“-Infektion. Klinische Zeichen können sehr diskret sein oder ganz fehlen. Eine präoperative Laboruntersuchung mit Erstellung eines Blutbildes sowie des C-reaktiven Proteins sind obligat. Bei unklarem Befund kann eine Leukozytenszintigrafie hilfreich sein. Manchmal ist auch eine direkte Punktion der Pseudarthrosenregion mit Gewinnung eines Aspirates und nachfolgender Anzüchtung hilfreich. Auch ein MRT kann hilfreich sein, um eine lokale Infektion auszuschließen. Bei betagten PatientInnen oder bei offensichtlicher Mangelernährung ist eine präoperative Testung von Serumprotein und des Albuminspiegels sinnvoll, und etwaige Defizite sind auszugleichen. 

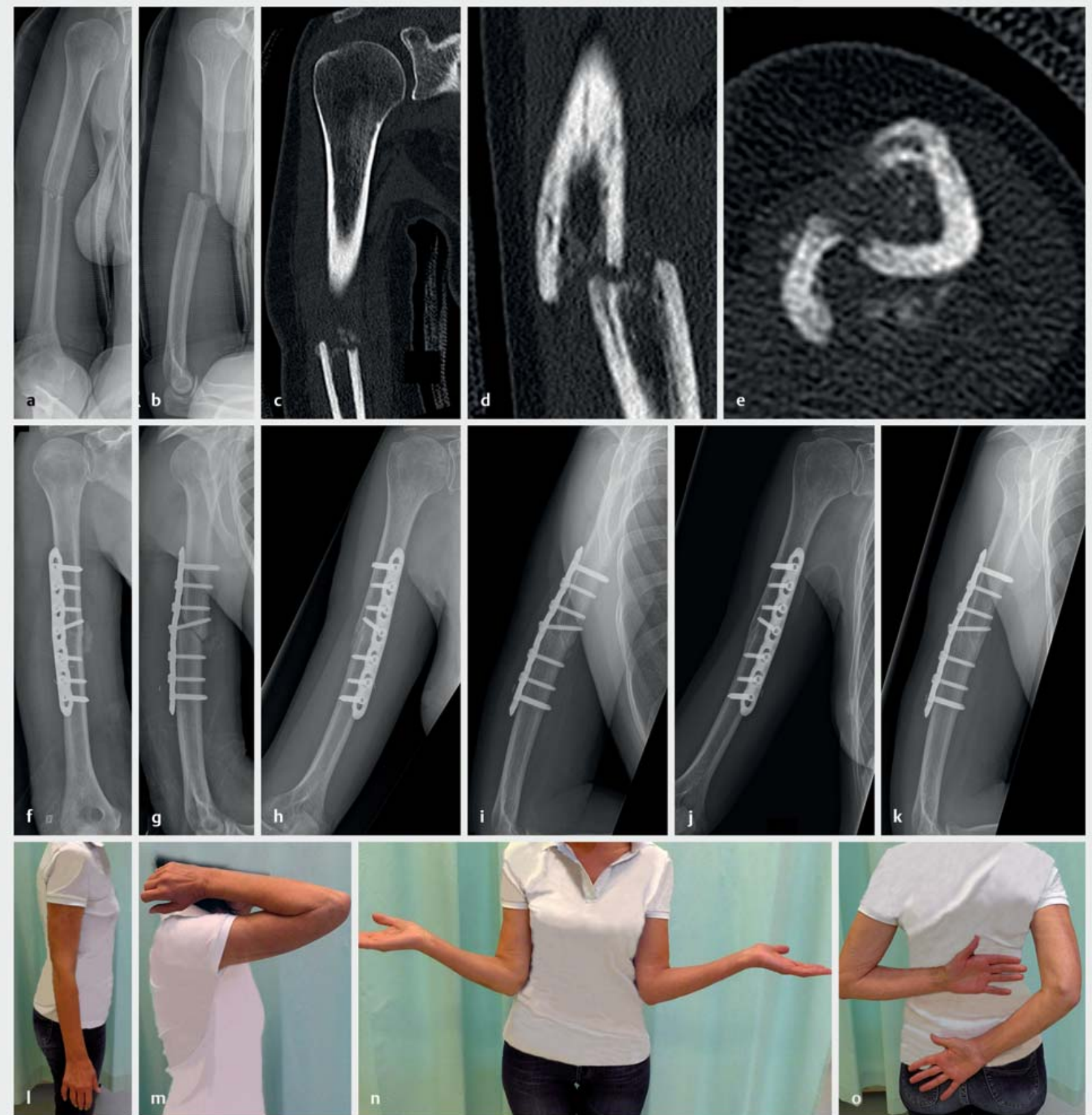

- Abb. 1 60-jährige Patientin 3 Monate nach konservativer Behandlung im Oberarm-Brace. Keine knöcherne Heilung. a, b Primäres Röntgen 3 Monate nach Fraktur - rechter Oberarm a.-p. und seitlich. c-e Primäres CT 3 Monate nach Humerusschaftfraktur. f, $g$ Röntgen postoperativ a.-p. und seitlich mit dorsaler Platte. h, i Röntgen 12 Wochen postoperativ. j, k Röntgen 1 Jahr nach OP - gute knöcherne Heilung. I-o Funktionelles Ergebnis 1 Jahr nach Versorgung.

Merke

Grundvoraussetzung für eine erfolgreiche Behandlung einer Pseudarthrose am Humerusschaft ist die intensive detaillierte Ursachenerforschung (lokal mechanisch oder biologisch/patientenspezifische Ursachen).
Die Behandlung symptomatischer Pseudarthrosen des Humerusschaftes ist i.d.R. operativ. Konservative Behandlung kommt bei PatientInnen mit begrenzten funktionellen Bedürfnissen bzw. durch etwaige Begleiterkrankungen stark erhöhtem OP-Risiko infrage. Einige neuere, nicht invasive Behandlungsoptionen sind derzeit in klini- 
scher Erprobung, wie z.B. die lokale Elektrostimulation, Ultraschallbehandlung und ganz besonders die lokale Stoßwellentherapie (ESWT). Erste Ergebnisse sind sehr vielversprechend und haben das Potenzial, in Zukunft in gewissen Fällen die operative Therapie abzulösen [12]. Für eine endgültige Bewertung fehlen aber noch hochwertige klinische Studien.

Ziel der operativen Therapie ist eine stabile Fixation mit der Möglichkeit zur frühfunktionellen Nachbehandlung, die Korrektur etwaiger Fehlstellungen und die Erzielung eines maximalen kortikalen Kontaktes und einer knöchernen Heilung. Hier gilt die Kompressionsplattenosteosynthese mit oder ohne Maßnahmen zur Verbesserung der Biologie (Dekortikation, autologe Knochentransplantation) als „Goldstandard“. Fehlstellungen werden am Humerus relativ gut kompensiert. Bis vor wenigen Jahren galt eine Fehlstellung von $20^{\circ}$ in jeder Richtung und ein Rotationsfehler von $30^{\circ}$ als Grenze für eine operative Intervention. Cresco et al. konnten 2016 jedoch zeigen, dass am Humerusschaft eine Varusfehlstellung wesentlich besser vertragen wird als eine Valgusfehlstellung [13]. Seither gilt für den Humerusschaft eine akzeptierte Grenze einer Fehlstellung von $<20^{\circ}$ Varus, $<15^{\circ}$ Valgus, Grenze sagittaler Deformität $<5^{\circ}$ und einer Rotationsfehlstellung $<30^{\circ}$. Der Zeitpunkt der chirurgischen Intervention bei verzögerter Heilung einer Humerusschaftfraktur ist nach wie vor Thema wissenschaftlicher Diskussion. Im Alltag ist dann, wenn nach 6-8 Wochen noch keine Zeichen einer knöchernen Heilung zu erkennen sind, höchste Aufmerksamkeit angezeigt und bei unverändertem Befund i.d.R. spätestens nach 10 Wochen das weitere Vorgehen mit dem Patienten zu besprechen.

Die Kompressionsplattenosteosynthese stellt das Standardverfahren in der Behandlung von Pseudarthrosen des Humerusschaftes dar. Sie wird entweder in Rückenlage, Bauchlage oder Beachchair-Lagerung über einen anterolateralen oder dorsalen Zugang durchgeführt. Das hauptsächlich verwendete Implantat ist, je nach individueller Knochendimension, die breite oder schmale Großfragment-LCP (4.5; LCP: Locking Compression Plate). Sie sollte eine ausreichende Länge aufweisen, sodass mindestens 6 oder besser 8 bikortikale Schrauben platziert werden können. Eine interfragmentäre Kompression wird angestrebt, entweder durch exzentrisches Besetzen der Plattenschrauben, durch eine interfragmentäre Zugschraube (durch die Platte oder plattenunabhängig) oder unter Verwendung des Plattenspanners ( $\downarrow$ Abb. 2). Bei hypertrophen Pseudarthrosen reicht die Stabilisierung allein i.d.R. aus, während bei schlechter lokaler Biologie eine Dekortikation (Anfrischen des Knochens mit einem Meißel) durchgeführt wird und eine autologe Spongiosaplastik, autologe Knochenspäne oder demineralisierte Knochenmatrix beigefügt wird. Bei atrophen Pseudarthrosen muss ein radikales Débridement durchgeführt werden, bis gesunder blutender Knochen freigelegt wird. Dazu kann auch eine leichte Verkürzung in Kauf genommen werden, die bei einer Länge bis zu 3$4 \mathrm{~cm}$ funktionell sehr gut toleriert wird. Der Markraum sollte in jedem Fall wieder eröffnet werden. Liegt bereits ein regelrechtes Neogelenk mit synovialer Auskleidung des Pseudarthrosenspaltes vor, so muss dieses mitsamt dem synovialen Gewebe reseziert werden. Die Resektion sollte unter ständiger Spülung zur Hitzevermeidung erfolgen. Auch über gute Erfahrungen mit der Cup-andCone-Technik wurde bereits berichtet [14]. Bei komplexen Situationen mit stark kompromittierter lokaler Biologie wurde auch die Anwendung von autologer Spongiosa in Kombination mit BMP-7 (OP-1, Osigraft) empfohlen [11]. Dies ist aber für die Pseudarthrosenbehandlung am Humerus nicht zugelassen und wird von uns daher im klinischen Alltag nicht verwendet.

\section{Merke \\ Die Kompressionsplattenosteosynthese mit einer LCP 4.5 mit Beilage von autologer Spongiosa stellt den „Goldstandard“ in der Behandlung von Pseudarthro- sen am Humerusschaft dar und führt in $98 \%$ zu einer knöchernen Heilung.}

Bei mangelnder Stabilität nach unilateraler Plattenosteosynthese, z. B. bei Pseudarthrosen im proximalen und distalen Schaftbereich, wodurch nicht ausreichend Schrauben im gelenknahen Fragment untergebracht werden können, ist die Doppelplattenosteosynthese eine gute Option. Damit können auch in schwierigen Situationen ausreichende Stabilität und gute Ausheilungsraten erzielt werden [15-17].

Pseudarthrosen bei liegendem Oberarmmarknagel kommen bei verbliebener Diastase, ausgebrochenen dislozierten Kortikalisfragmenten oder zusätzlicher Trümmerzone vor. Als klassische Therapieoptionen kommen ein Nagelwechsel mit zusätzlichem Aufbohren des Markraumes oder nach Nagelentfernung der Umstieg auf eine Plattenosteosynthese mit zusätzlicher Spongiosaanlagerung infrage [18]. Das reine Aufbohren mit Nagelwechsel führt aber zu geringeren Ausheilungsraten als der Nagelwechsel mit Dekortikation und Spongiosaanlagerung, ebenso wie der Verfahrenswechsel auf eine Platte mit additiver autologer Spongiosaanlagerung [19-21]. Eine weitere Therapieoption stellt die additive Plattenosteosynthese ohne Entfernung des Marknagels dar ( $\bullet$ Abb. $\mathbf{3}$ ). Diese kann als additive gerade Platte oder als Wellenplatte in Kombination mit einer Dekortikation und Spongiosaplastik angewandt werden und führt in einem hohen Prozentsatz zu einer knöchernen Heilung [22].

Eine große Herausforderung an das behandelnde Team stellen Infektpseudarthrosen dar. Ein sorgfältiges Débridement und die Entfernung etwaiger Implantate sowie aller infizierter Gewebeanteile und Sequester sind von größter Wichtigkeit. Je nach der entstandenen Defekt- 

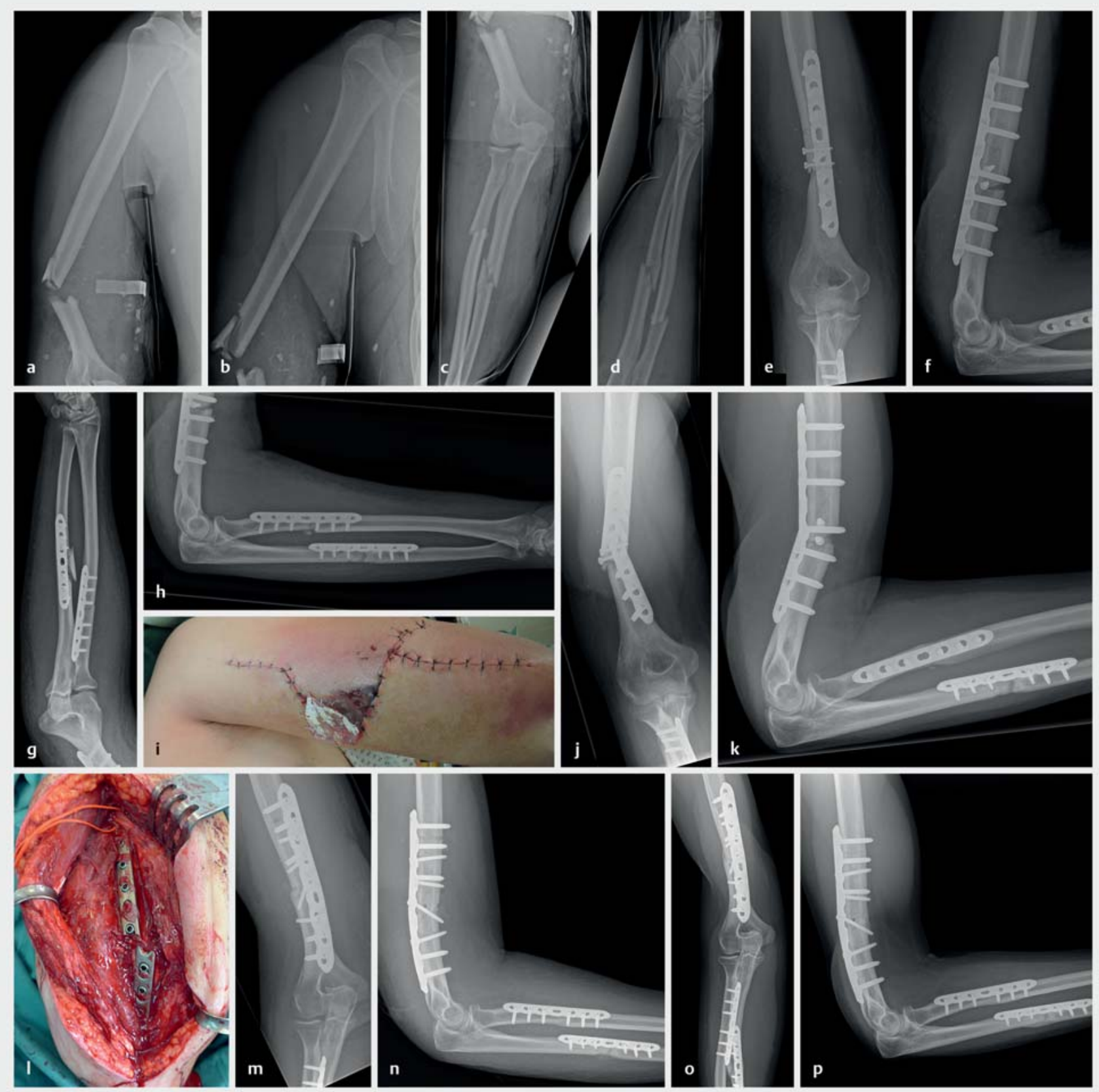

- Abb. 2 37-jähriger Mann. Motorradsturz mit offener Fraktur des Humerus und Schaftfraktur der Vorderarmknochen. Primäre Radialisparese. Weichteilschaden. a-d Primäre Röntgenbilder nach dem Unfall - rechter Oberarm und Unterarm. e-i Postoperative Röntgenbilder nach Débridement, Plattenosteosynthese und N.-radialis-Rekonstruktion. j-I Röntgen und intraoperativer Situs 6 Monate nach der Osteosynthese - Plattenbruch, Fraktur nicht geheilt. m, n Postoperative Röntgenbilder nach Reosteosynthese. o, p Röntgenkontrollen 6 Monate nach der Reoperation die Fraktur ist gut knöchern geheilt.

größe und den detektierten Keimen kann ein einzeitiges oder zweizeitiges Verfahren notwendig sein. Beim einzeitigen Vorgehen wird unter Verkürzung eine Stabilisierung mittels Plattenosteosynthese durchgeführt, während beim zweizeitigen Vorgehen ein Fixateur externe zur temporären Stabilisierung zur Anwendung kommt und der Defekt mit einer Plombe aus antibiotikahaltigem Knochenzement aufgefüllt wird. Nach Infektsanierung wird (nach durchschnittlich nach 6-8 Wochen) unter Belassen der umgebenden Membran der Spacer entfernt und die Defekthöhle mit Spongiosachips aufgefüllt (Masquelet-Technik) [11,23, 24]. Bei größeren Defekten kann 

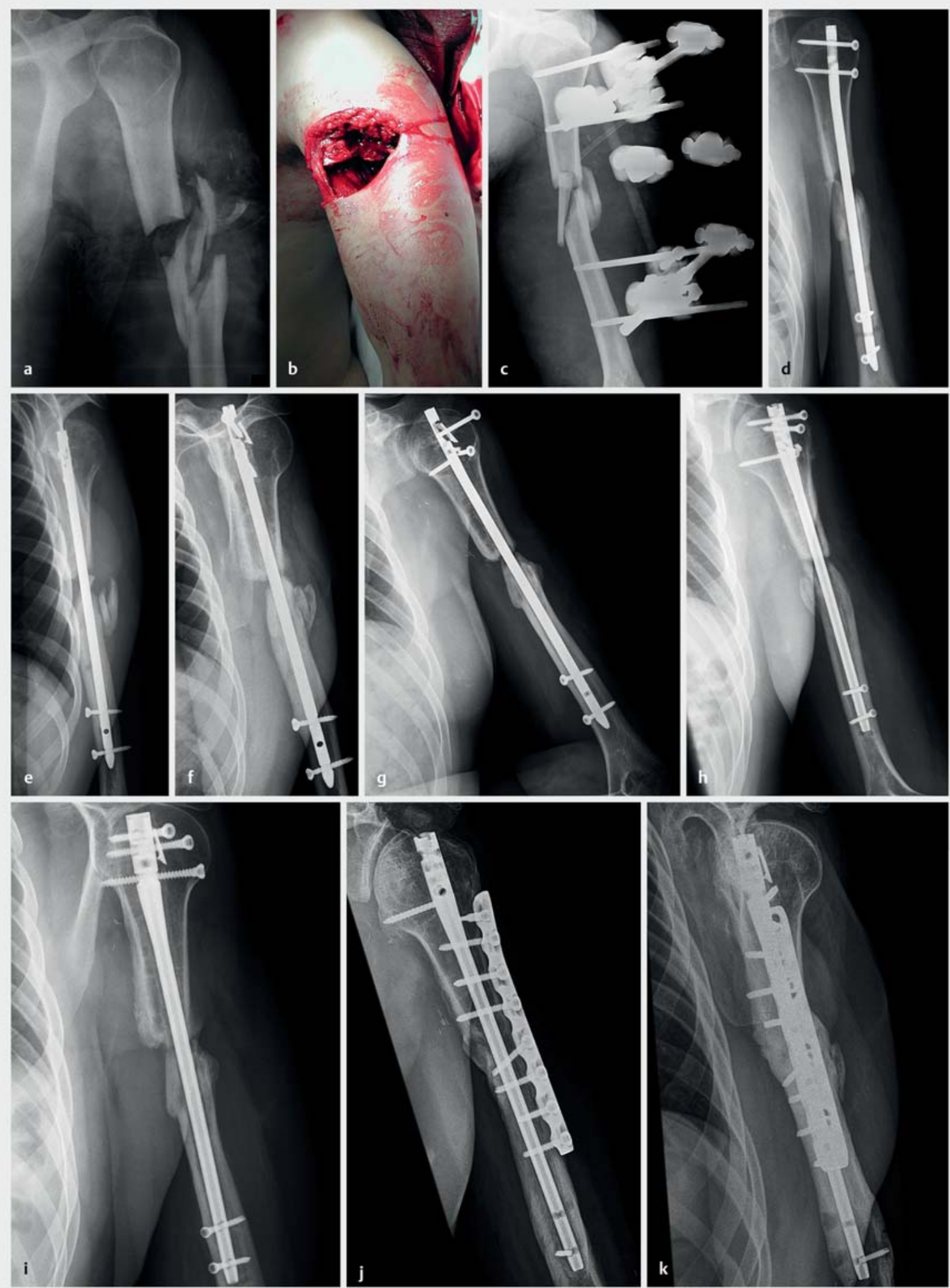

- Abb. 3 24-jähriger Mann. Motorradunfall, Kollision mit einem Traktor. Polytraumatisiert. Offene Fraktur linker Humerus mit primärer Radialisparese. a, b Primäres Röntgen linker Oberarm, und lokaler Weichteilsitus. c Akutversorgung mit Débridement und Fixateur externe (Damage Control Surgery). d, e Postoperatives Röntgen nach Marknagelung. f, g Vier Jahre nach der Erstversorgung - Defektpseudarthrose und Lockerung des Marknagels. h, i Reoperation mit neuem Marknagel und Spongiosabeilage. j, k Fünf Jahre nach Erstversorgung keine knöcherne Heilung und daher Reoperation mit additiver Platte, Dekortikation und nochmaliger Spongiosaplastik (RIA). 
auch im Einzelfall ein Segmenttransport (Ilizarov-Methode) notwendig sein [25].

Größere Knochendefekte können auch mit kortikospongiösen Autografts, Allografts oder gefäßgestielten Knochengrafts überbrückt werden. Allografts können intramedullär oder als Zusatz zu einer Plattenosteosynthese appliziert werden [26]. Als gefäßgestielte Autografts kommen die gefäßgestielte Fibula, Späne aus dem Femurkondylus, der Skapula oder aus dem Beckenkamm infrage $[27,28]$. Damit können auch bei großen Knochendefekten und eingeschränkter lokaler biologischer Kompetenz gute Heilungsergebnisse erzielt werden.

\section{Ergebnisse}

Im Zeitraum von 2000 bis Ende 2018 hatten wir im eigenen Patientenkollektiv 80 Pseudarthrosen im Bereich des Oberarmes zu behandeln. Davon waren 28 im proximalen Anteil, 30 im Schaftbereich und 22 distal. Das Durchschnittsalter betrug bei den Schaftpseudarthrosen 63,3 Jahre, wobei eine deutliche bimodale Altersverteilung zu erkennen war (min. 28/max. 94). Ein großer Teil der Schaftpseudarthrosen wurde mit einer Plattenosteosynthese mit und ohne Spongiosaplastik versorgt. Obwohl verschiedene Behandlungsmethoden zur Heilung einer Oberarmschaftpseudarthrose zur Verfügung stehen, hat doch im Einklang mit der Literatur die Kompressionsplattenosteosynthese mit und ohne Beigabe von autologer Spongiosa die besten Ergebnisse erbracht. In großen Kohorten konnte durch eine Plattenosteosynthese mit Kompression und Spongiosaplastik in 98\% eine knöcherne Heilung erzielt werden [29,30]. Ohne beigelegte Spongiosa ergab die Plattenosteosynthese eine Heilungsrate von 95\% und der Marknagel ohne Spongiosa 66\%. Die Gesamtkomplikationsrate lag bei der Plattenosteosynthese mit Spongiosa bei $12 \%$ und bei Verwendung des Marknagels zwischen 8 und 15\%. Alternative Behandlungsmaßnahmen haben bei richtiger Indikationsstellung ebenfalls in einem hohen Ausmaß sehr gute Ergebnisse und rasche Heilung erbracht. Bei genauer präoperativer Analyse und personenspezifischer Behandlung unter Beachtung des Diamond-Konzeptes kann i.d.R. eine verlässliche Ausheilung der Pseudarthrosen am Humerusschaft erreicht werden. Die Kompressionsplattenosteosynthese mit Spongiosabeigabe stellt nach wie vor den „Goldstandard“ dar.

\section{Distaler Humerus}

Distale Humerusfrakturen sind mit 2\% aller Frakturen eher seltene Verletzungen, stellen aber aufgrund ihrer häufigen Komplexität doch große Herausforderungen an den Behandler. Die primäre Behandlung frischer Frakturen ist i.d. R. die offene Rekonstruktion und stabile Doppelplattenosteosynthese. Trotz einer deutlichen Verbesserung der Operationstechnik liegt die Pseudarthrosenrate nach distalen Humerusfrakturen auch heute noch bei 6-25\%. Häufig ist die suprakondyläre Region betroffen; gelegentlich finden sich intraartikuläre Pseudarthrosen, die nicht selten von unzureichend behandelten Kondylenfrakturen im Kindes- bzw. Jugendalter herrühren. Der häufigste Grund ist eine unzureichende Erstbehandlung, sei es eine inadäquate Ruhigstellung oder Fixationstechnik. Trümmerzonen, schlechte Knochenqualität oder sehr tief liegende komplexe Frakturformen, besonders bei geriatrischen PatientInnen, oder offene Frakturen sowie Infektionen begünstigen die Entstehung einer Pseudarthrose. Allgemeine Faktoren, die das Ausbleiben einer Heilung begünstigen, sind Rauchen, immunsuppressive Therapie und gewisse andere Medikamente wie NSAR, Diabetes oder eine schlechte Patientencompliance. Suprakondyläre Humeruspseudarthrosen sind meist mit einer Kontraktur des Ellbogengelenkes vergesellschaftet, wodurch es zu vermehrten Bewegungen in der Pseudarthrose kommt. Durch diese pathologische Bewegung kommt es auch zu einer oft erheblichen Schädigung des Knochens. Häufig kommt es auch zu einer Irritation und Beeinträchtigung des N. ulnaris. Während intraartikuläre Pseudarthrosen, die im Kindes- oder Jugendalter nach Kondylenfrakturen entstehen, oft lange kompensiert werden können und erst später meist durch die Neuropathie des N. ulnaris Beschwerden verursachen, führen suprakondyläre Pseudarthrosen beim Erwachsenen sehr früh zu Schmerzen, Funktionsverlust und Instabilität („flail elbow“).

\section{Behandlung}

Präoperativ ist zur genauen Planung neben einem Nativröntgen in mehreren Ebenen ein CT mit 3-D-Rekonstruktion und evtl. die Anfertigung eines gedruckten Knochenmodells sinnvoll. Eine Infektion muss ausgeschlossen werden. Ziel ist eine stabile Osteosynthese mit einer Doppelplattenosteosynthese und ein guter Knochenkontakt. Nach Neurolyse des N. ulnaris muss zuvor der Markraum auf beiden Seiten eröffnet werden und evtl. vorhandenes Osteosynthesematerial entfernt werden. Besteht ein Knochendefekt, so kann der Schaft verkürzt und unter Kompression in den distalen Gelenkblock eingefalzt werden. Beide werden dann durch eine Doppelplattenosteosynthese stabil miteinander verbunden. Eine Anlagerung von autologen Knochenspänen erhöht die Chance auf eine ungestörte Knochenheilung [31]. Fehlpositionen innerhalb der Gelenkfläche sollten korrigiert und möglichst stufenlos fixiert werden. Ein ausgedehntes Kapselrelease am Ellbogengelenk ist obligatorisch.

Pseudarthrosen bei älteren Patienten oder unter erheblicher Mitbeteiligung der Gelenkflächen, wenn eine stabile anatomische Rekonstruktion nicht möglich scheint, sind gute Indikationen für eine teilverblockte Ellbogentotalendoprothese [31] ( $\triangleright$ Abb. 4). Wir bevorzugen einen dorsalen Zugang mit einer trizepserhaltenden Operationstechnik. Eine Resektion der meist verdickten kontrakten Gelenkkapsel ist notwendig. In der Regel führt dies zu 

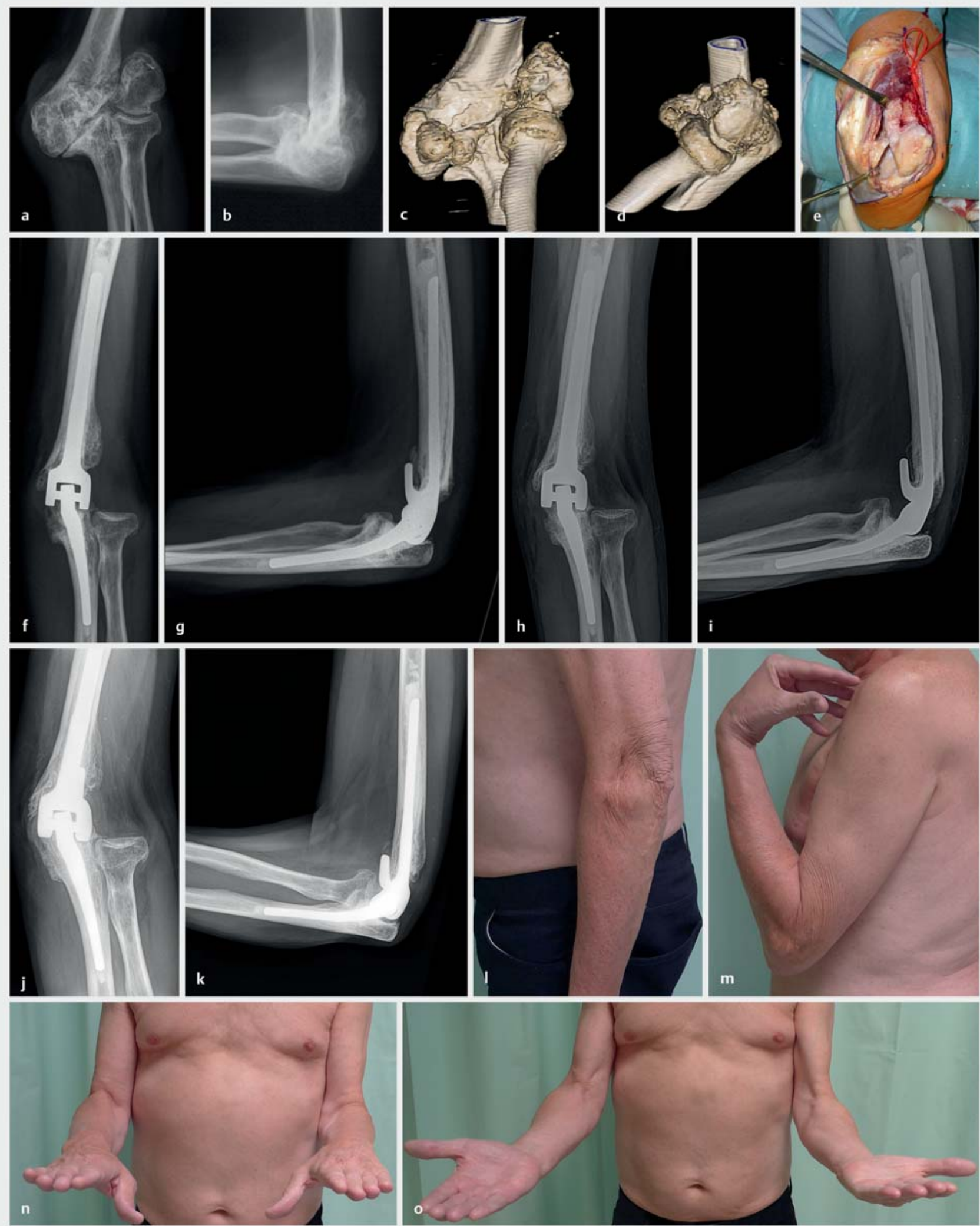

- Abb. 4 67-jähriger Pensionist. 50 Jahre nach Sturz mit Verletzung des linken Ellbogengelenkes. Jetzt dekompensierte Pseudarthrose, mit starken Schmerzen und zunehmender Bewegungseinschränkung. a-d Primäres Röntgen und CT 50 Jahre nach Verletzung - Pseudarthrose distaler Humerus. e Intraoperativer Situs. $\mathbf{f}, \mathbf{g}$ Postoperatives Röntgen. h, i Röntgen 1 Jahr nach Implantation einer Ellbogenprothese. j-o Röntgen und Funktion 7 Jahre nach Implantation der Ellbogentotalendoprothese. 
einem stabilen, sehr gut beweglichen und gut kontrollierbaren Arm. Eine Belastungslimitierung muss dem Patienten aufgetragen werden, um eine Überlastung der Prothese zu vermeiden.

\section{Merke}

Die Behandlung der Pseudarthrose am distalen Humerus besteht meist in einer neuerlichen Doppelplattenosteosynthese mit Kompression an den beiden Pfeilern in Kombination mit einer autologen Knochenspananlagerung. Bei geriatrischen Patienten, oder wenn eine stabile Rekonstruktion nicht möglich erscheint, ist die teilverblockte Ellbogentotalendoprothese eine gute Rettungsoperation.

\section{Ergebnisse}

Generell sind die Ergebnisse nach Reosteosynthese von Pseudarthrosen am distalen Humerus gut, sofern eine knöcherne Heilung erzielt werden kann. Da die Heilung im Vordergrund steht, sind oft sekundäre Eingriffe zur Wiederherstellung der Beweglichkeit angezeigt. Die bei unseren 22 PatientInnen erzielten Bewegungsumfänge und Ausheilungsraten sind äußerst zufriedenstellend. Die Komplikationsraten sind im Einklang mit der Literatur akzeptabel. Hier werden Infektionen und Nervenschädigungen (wenn auch nur temporär) angegeben [32-34]. Die teilverblockte Ellenbogentotalendoprothese erlaubt eine Wiederherstellung der Beweglichkeit und Schmerzfreiheit, jedoch um den Preis einer verminderten Belastbarkeit $[35,36]$. Dies hat in unserem gut selektionierten Patientengut zu einer hohen Patientenzufriedenheit geführt.

\section{Schlussfolgerung}

Symptomatische Pseudarthrosen im Bereich des Humerusschaftes und auch am distalen Humerus bedürfen einer operativen Behandlung. Meist führt die Plattenosteosynthese mit interfragmentärer Kompression und zusätzlicher autologer Spongiosaanlagerung zu einer guten soliden Heilung. Bei nicht rekonstruierbaren distalen Humeruspseudarthrosen kann die Ellenbogentotalendoprothese verbunden mit einem umfassenden Weichteilrelease zu guten Ergebnissen führen.

\section{Interessenkonflikt}

Der Autor gibt an, keine Interessenkonflikte mit dem Inhalt dieses Artikels zu haben. Er oder seine Institution bezieht keinerlei finanzielle Zuwendungen von Firmen, deren Produkte in dieser Arbeit genannt werden.

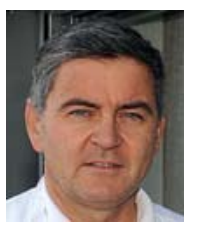

\section{Michael Plecko}

Prim. Dr. med., Ärztlicher Direktor des Unfallkrankenhauses Steiermark der AUVA, Standort Graz, Lehrkrankenhaus der MEDUNI Graz

Korrespondenzadresse

Prim. Dr. med. Michael Plecko

Unfallkrankenhaus Steiermark der AUVA

Lehrkrankenhaus der MEDUNI Graz

Göstingerstraße 24

8020 Graz

Österreich

Tel.: + 43/(0)5939343201

Fax: + 43/(0)5939343205

michael.plecko@auva.at

\section{Literatur}

[1] Sarmiento A, Kinman PB, Heckman JD et al. Functional bracing of fractures of the shaft of the humerus. J Bone Joint Surg Am 1977; 59: 596-601

[2] Zagorski JB, Latta LL, Zych GA et al. Diaphyseal fractures of the humerus. Treatment with prefabricated braces. J Bone Joint Surg Am 1988; 70: 607-610

[3] Athwal G. Distal Humerus Fractures. In: Court-Brown CM, Heckman JD, McQueen MM, Ricci WM, Tornetta P III, McKee $M D$, eds. Rockwood and Green's Fractures in Adults. 8th ed. Vol. 1. Philadelphia: Wolters Kluwer Health; 2015: 1229-1286

[4] Jupiter JB, von Deck M. Ununited humeral diaphysis. J Shoulder Elbow Surg 1998; 7: 644-653

[5] Ali E, Griffith D, Obi N et al. Nonoperative treatment of humeral shaft fractures revisited. J Shoulder Elbow Surg 2015; 24: 210-214

[6] Denard A jr., Richards JE, Obremskey WT et al. Outcome of nonoperative vs. operative treatment of humeral shaft fractures: a retrospective study of 213 patients. Orthopedics 2010. doi:10.3928/01477447-20100625-16

[7] Matsunaga FT, Tamaoki MJ, Matsumotot MH et al. Minimally invasive osteosynthesis with bridge plate versus a functional brace for humeral shaft fractures: a randomized controlled trial. J Bone Joint Surg Am 2017; 99: 583-592

[8] Harkin FE, Large RJ. Humeral shaft fractures: union outcomes in a large cohort. J Shoulder Elbow Surg 2017; 26: 1881-1888

[9] Papasoulis E, Drosos GI, Ververidis AN et al. Functional bracing of humeral shaft fractures: a review of clinical studies. Injury 2010; 41: e21-e27

[10] Giannoudis PV, Einhorn TA, Marsh D. Fracture healing: the diamond concept. Injury 2007; 38 (Suppl. 4): S3-S6

[11] Miska M, Findeisen S, Tanner M et al. Treatment of nonunions in fractures of the humeral shaft according to the Diamond Concept. Bone Joint J 2016; 98-B: 81-87

[12] Willems A, van der Jagt OP, Meuffels DE. extracorporal shock wave treatment for delayed union and nonunion fractures: a systematic review. J Orthop Trauma 2019; 33: 97-103

[13] Crespo AM, Konda SR, De Paolis A et al. Posttraumatic malalignment of the humeral shaft: challenging the existing paradigm. J Orthop Trauma 2016; 30: e48-e52 
[14] Nickel BT, Klement MR, Richard M] et al. Closing the gap: a noval technique for humeral shaft nonunions using cup and cone reamers. Injury 2016; 47 (Suppl. 7): S40-\$43

[15] Prasarn ML, Achor T, Paul O et al. Management of nonunions of the proximal humeral diaphysis. Injury 2010; 41: 12441248

[16] Rubel IF, Kloen P, Campbell D et al. Open reduction and internal fixation of humeral nonunions: a biomechanical and clinical study. J Bone Joint Surg Am 2002; 84: 1315-1322

[17] Metikala S, Bhogadi P. Orthogonal double plating and autologous bone grafting of postoperative humeral shaft nonunion a rare case report and review of literature. J Orthop Case Rep 2015; 5: 50-53

[18] Lin CL, Fang CK, Chiu FY et al. Revision with dynamic compression plate and cancellous bone graft for aseptic nonunion after surgical treatment of humeral shaft fracture. J Trauma 2009; 67: 1393-1396

[19] Lin J, Chiang H, Hou SM et al. Open exchange locked nailing in humeral nonunions after intramedullary nailing. Clin Orthop Relat Res 2003; (411): 260-268

[20] Mc Kee MD, Miranda MA, Riemer BL et al. Management of humeral nonunion after the failure of locking intramedullary nails. J Orthop Trauma 1996; 10: 492-499

[21] Flinkkila T, Ristiniemi J, Hamalainen M. Nonunion after intramedullary nailing of humeral shaft fractures. J Trauma 2001; 50: $540-544$

[22] Gessmann J, Königshausen M, Coulibaly MO et al. Anterior augmentation plating of aseptic humeral shaft nonunions after intramedullary nailing. Arch Orthop Trauma Surg 2016; 136: 631-638

[23] Masquelet AC, Begue T. The concept of induced membrane for reconstruction of long bone defects. Orthop Clin North Am 2010; 41: 27-37

[24] Taylor BC, French BG, Fowler TT et al. Induced membrane technique for reconstruction to manage bone loss. J Am Acad Orthop Surg 2012; 20: 142-150

[25] Kiran M, Jee R. llizarov's method for treatment of nonunion of diaphseal fractures of the humerus. Indian J Orthop 2010; 44: 444-447

[26] Vidyadhara S, Vamsi K, Rao SK et al. Use of intramedullary fibular strut graft: a novel adjunct to plating in the treatment of osteoporotic humeral shaft nonunion. Int Orthop 2009; 33 : 1009-1014
[27] Muramatsu K, Doi K, Ihara K et al. Recalcitrant posttraumatic nonunion of the humerus. 23 patients reconstructed with vascularized bone graft. Acta Orthop Scand 2003; 74: 95-97

[28] Kaminiski A, Bürger H, Müller E]. Free vascularised corticoperiosteal bone flaps in the treatment of non-union of long bones: an ignored opportunity. Acta Orthop Belg 2008; 74: 235-239

[29] Peters RM, Claessen FM, Doornberg JN et al. Union rate after operative treatment of humeral shaft nonunion - a systematic review. Injury 2015; 46: 2314-2324

[30] Babhulkar S, Babhulkar S, Vasudev A. Recalcitrant aseptic atrophic non-union of the shaft of the humerus after failure of surgical treatment: management by excision of non-union, bone grafting and stabilization by LCP in different modes. Injury 2017; 48 (Suppl. 2): S33-S43

[31] Sanchez-Sotelo J. Nonunion and Malunion of distal Humerus Fractures. In: Morrey BF, Sanchez-Sotelo J, Morrey ME, eds. The Elbow and its Disorders. Philadelphia: Elsevier; 2018: 501-509

[32] Donders JCE, Lorich DG, Helfet DL et al. Surgical technique: treatment of distal humerus nonunion. HSS J 2017; 13: 282291

[33] Helfet DL, Kloen P, Anand N et al. Open reduction and internal fixation of delayed unions and nonunions of fractures of the distal part of the humerus. J Bone Joint Surg Am 2003; 85: $33-40$

[34] Ring D, Gulotta L, Jupiter JB. Unstable nonunion of the distal part of the humerus. J Bone Joint Surg Am 2003; 85: 1040 1046

[35] Sanchez-Sotelo J, Morrey BF. Linked elbow replacement: a salvage procedure for distal humeral nonunion. Surgical technique. J Bone Joint Surg Am 2009; 91 (Suppl. 2): S200-S212

[36] Cil A, Veillette C], Sanchez-Sotelo J et al. Linked elbow replacement: a salvage procedure for distal humeral nonunion. J Bone Joint Surg Am 2008; 90: 1939-1950

Bibliografie

DOI https://doi.org/10.1055/a-0898-6681

OP-JOURNAL 2019; 35: 274-282 @ Georg Thieme Verlag KG Stuttgart · New York ISSN 0178-1715 